\title{
Risk of dementia and death in community-dwelling older men with bipolar disorder
}

\author{
Osvaldo P. Almeida, Kieran McCaul, Graeme J. Hankey, Bu B. Yeap, Jonathan Golledge \\ and Leon Flicker
}

\section{Background}

Bipolar disorder has been associated with cognitive decline, but confirmatory evidence from a community-derived sample of older people is lacking.

\author{
Aims \\ To investigate the 13-year risk of dementia and death in \\ older adults with bipolar disorder.
}

\section{Method}

Cohort study of 37768 men aged 65-85 years. Dementia (primary) and death (secondary), as recorded by electronic record linkage, were the outcomes of interest.

\section{Results}

Bipolar disorder was associated with increased adjusted hazard ratio (HR) of dementia $(\mathrm{HR}=2.30,95 \% \mathrm{Cl} 1.80-2.94)$. The risk of dementia was greatest among those with
$<5$ years of history of bipolar disorder or who had had illness onset after 70 years of age. Bipolar disorder was also associated with increased mortality $(\mathrm{HR}=1.51,95 \% \mathrm{Cl}$ 1.28-1.77). Competing risk regression showed that bipolar disorder was associated with increased hazard of death by suicide, accidents, pneumonia or influenza, and diseases of the liver and digestive system.

\section{Conclusions}

Bipolar disorder in later life is associated with increased risk of dementia and premature death.

\section{Declaration of interest}

None.

\section{Copyright and usage}

(c) The Royal College of Psychiatrists 2016.
Evidence accumulated over the past decade has raised questions about the commonly held notion that bipolar disorder has a benign course. A longitudinal study of 71 adolescents admitted with their first ever mixed or manic episode found that, over 12 months, only $41 \%$ of them had achieved full functional recovery, even though $86 \%$ were free from affective symptoms. ${ }^{1}$ There are growing concerns that bipolar disorder may be associated with cognitive deficits even during euthymic periods. For example, Mora and colleagues examined 28 adults with bipolar disorder and 26 healthy controls (mean age 41 years). ${ }^{2}$ After a follow-up period of 6 years, participants with euthymic bipolar disorder had experienced a significant decline in tests of executive functioning, processing speed and verbal memory compared with healthy controls. ${ }^{2}$ Recent meta-analyses of neuropsychological studies of young and elderly euthymic people with bipolar disorder reported results consistent with those findings. ${ }^{3,4}$ In addition, there is evidence that the level of functional impairment in adults with bipolar disorder is correlated with the severity of cognitive deficits, ${ }^{5}$ which seem particularly pronounced among those with illness onset in mid or late life. ${ }^{6}$ It remains unclear, at this stage, whether these deficits are progressive in nature and ultimately lead to an increase in the risk of dementia as people with bipolar disorder age. Kessing \& Andersen used the Danish Health Register to investigate the risk of dementia among 4248 adults (mean age 57 years) admitted to hospital with a primary diagnosis of bipolar disorder between 1970 and $1986 .^{7}$ Follow-up for dementia ended on 31 December 1999. They found that the risk of dementia increased, on average, $6 \%$ for every additional affective episode that required admission to hospital. ${ }^{7}$ They also reported, in a separate study, that the probability of dementia over time was more strongly associated with the diagnosis of bipolar disorder than with unipolar depression, schizophrenia or neurosis. ${ }^{8}$ A nested case-control study using data from the Taiwanese National Health Insurance Research Database found that bipolar disorder was associated with a four-fold increase in the odds of dementia, including dementia with onset before the age of 65 years. ${ }^{9}$ It remains unclear, however, whether the relationship between bipolar disorder and dementia is truly causal. Furthermore, existing data suggest that adults with bipolar disorder die prematurely, ${ }^{10,11}$ and the same seems to occur in people with bipolar disorder who reach older age. ${ }^{12}$ Mortality data on middle-aged and young older adults have been reported elsewhere, ${ }^{13}$ but information about the causes of death for people with bipolar disorder who reach older age has not been established, so it is unclear whether some of these deaths could be prevented.

The present cohort study aimed to investigate the risk of dementia and death among community-dwelling older adults. We also sought to determine the causes of death in the population and among older people with bipolar disorder.

\section{Method}

\section{Study design and setting}

The Health in Men Study (HIMS) is a cohort study of a community-representative sample of older Western Australian men that started in 1996. Follow-up data were censored on 30 June 2009, which is the last date for which data on cause-specific mortality are available.

\section{Participants}

Men aged 65-85 years were identified using an electronic copy of the Western Australian electoral roll (enrolment to vote is compulsory for all Australian adults). We received a list with the contact details of 49801 men, the entire population of men in this age group living in the Perth metropolitan region in 1995. Of those, 1839 had died by the time the study started and another 9482 were not selected for participation because they were living outside the immediate Perth metropolitan region. Of the remaining 38480 men, 407 were excluded because they had a 
recorded diagnosis of dementia (see below), and another 305 were younger than 65 years. This left a total study sample of 37768 older men who were alive and free of dementia by 2 April 1996.

This study was approved by the ethics committees of the University of Western Australia and of the Department of Health of Western Australia, as well as by the Legal Data Custodian.

\section{Outcomes: dementia and death}

The primary outcome of interest was the diagnosis of dementia recorded in the Western Australian Data Linkage System (WADLS). WADLS links information for all contacts with out-patient and in-patient mental health services in Western Australia, hospital morbidity data, as well as cancer and death registries. ${ }^{14}$ From 1 January 1966 to 31 December 1978 diagnostic codes followed the ICD $-8 ;{ }^{15}$ from 1 January 1979 to 30 June 1999 , ICD-9 $9^{16}$ codes were used, and from the 1 July 1999 all codes followed the ICD- $10^{17}$ system. The following codes identified participants with dementia: 290, 294.1, 294.2, 331.0, 331.1, 331.2, 331.82, F00, F01, F02, F03, G30, G31.0, G31.1, and G31.83. In addition, we systematically scanned the text of death records for the words 'dementia', 'Alzheimer', 'Pick' and 'Lewy' to identify men who had a recorded diagnosis of dementia at the time of their death. The diagnoses of dementia were recorded between 2 April 1996 and 30 June 2009.

We also examined the death records of WADLS (secondary outcome of interest). The causes of death were grouped into 12 categories: suicide (ICD codes: E950-E959, X60-X84, Y870 and U03), accident (ICD codes: E80-E929, V00-V999, W00-W199, Y85-Y859), infection (ICD codes: 00-139, 771.3, A00-A99, B00-B99), cancer (ICD codes: 140-208, C00-C97), diseases of the heart (ICD codes: 390-398, 402, 404, 410-429, I00-I09, I11, I13, I20-I51), diseases of the brain (ICD codes: 30-32, 35-37, 290-294, 320-337, 340-349, 430-434, 436-438, I60-I69, G00-G14, G20-G26, F00-F09), vascular diseases that exclude heart conditions and strokes (ICD codes: 401, 403, 440-448, I10, I12, I70-I78), pneumonia or influenza (ICD codes: 480-487, J09-J18), respiratory diseases (ICD codes: 460-466, 470-478, 490-496, 507-519, J00-J47, J60-J99), diseases of the digestive system (including liver) (ICD codes: 520-537, 540-543, 550-553, 555-589, K00-K99), diseases of the kidneys (ICD codes: 580-589, N0-N7, N17-N19, N25-N27), and other causes of death (remaining ICD codes). Mortality data were available for the period between 2 April 1996 and 30 June 2009.

\section{Exposure at study entry: bipolar disorder}

We retrieved information about past diagnosis of bipolar disorder from the WADLS, which had to have been recorded prior to 2 April 1996 according to the following ICD codes: 296.1 and 296.3 (ICD-8), and 296.0, 296.1, 296.4, 296.5, 296.6, 296.7, 296.80 and 296.81 (ICD-9). We considered that the date of the first ever episode of bipolar disorder was the same as the date of the first ever contact with the health services with one of these diagnoses (out-patient or in-patient). If a depressive episode had been recorded prior to the episode of mania or mixed state, then the date of the depressive episode was considered the date of onset of the bipolar disorder. The ICD codes used to identify depressive episodes were: 296.0 and 300.4 (ICD-8), and 296.2, 296.3, 311 and 300.4 (ICD-9). The earliest mental health morbidity records available were dated 1 January 1966.

\section{Other study measures}

Other variables included date of birth, alcohol use disorder (ICD codes: 291, 303 and 305.0) or substance use disorder (ICD codes: 292, 304 and 305 - excluding 305.0). Past studies have shown that WADLS yields accurate diagnoses for severe mental disorders. ${ }^{18}$
We calculated the age of participants as the difference, in years, between the date at study entry and the date of birth. The duration (in years) of the bipolar episode was calculated as the difference between the date at the beginning of the follow-up period and the date of the first ever recorded affective episode of bipolar disorder.

\section{Statistical methods}

We used the statistical package Stata/IC 14.0 to manage and analyse the data (StataCorp LP, 2015). Descriptive statistics summarised categorical data as count and proportions (\%), and continuous variables as mean, range and standard deviation of the mean (s.d.). We used the proportion command of Stata to estimate the prevalence of severe mental disorders (95\% confidence intervals ( $95 \%$ CIs) were calculated using a logit transformation that allows for the estimation of the standard error of the proportion). We employed logistic regression to estimate the odds ratio (OR) of the association between bipolar disorder and age strata and prevalent alcohol and substance use disorder, and Cox regression to estimate the hazard ratio ( $\mathrm{HR}$, and $95 \% \mathrm{CI}$ ) of dementia and death associated with bipolar disorder. The Cox regression models were adjusted for age, with the date of birth of participants set as the origin, the beginning of follow-up as the date of invitation, and the date of first ever diagnosis of dementia or death as the time of the event. Data were censored on 30 June 2009. Survival data were plotted using the Kaplan-Meier survivor function, and the cumulative hazard using the Nelson-Aalen approach. We supplemented these analyses with a competing risk Cox regression model using death as an outcome competing with dementia. The risk estimates, in this case, are expressed as subhazard ratios (SHRs) and respective 95\% CI. We used the stptime command of Stata to calculate the age-standardised annual rate of dementia and of death for people with and without bipolar disorder. We repeated this analytical approach after stratifying participants with bipolar disorder according to their age at the time of onset of illness (40s or earlier, 50s, 60s, 70s or 80s) and according to the duration of the disorder ( $<5$ years, $5-14$ years, $>15$ years). Finally, we investigated the association between the diagnosis of bipolar disorder (and age at onset/duration of the disorder) and cause-specific mortality using age-adjusted competing risk Cox regression. Alpha was set at 0.05 and all tests reported are two-tailed.

\section{Results}

The sample consisted of 37768 men aged $65-85$ years (mean 72.5 , s.d. $=4.6)$, of whom $256(0.7 \%, 95 \%$ CI $0.6-0.8)$ had a recorded diagnosis of bipolar disorder at study entry. The age distribution of men with and without bipolar disorder was similar $(t=1.27$, d.f. $=37766, P=0.203)$. Table 1 summarises their characteristics.

Overall, $13 \%$ of participants $(n=4925)$ developed dementia, which was present in $65(25.4 \%, 95 \%$ CI $20.4-31.1)$ of the 256 men with bipolar disorder. Bipolar disorder was associated with higher age-adjusted hazard of dementia ( $\mathrm{HR}=2.58,95 \%$ CI 2.02-3.30) (Fig. 1), and this association remained largely unchanged after additional adjustment for history of alcohol and substance use disorder $(\mathrm{HR}=2.30,95 \%$ CI 1.80-2.94). Competing risk Cox regression yielded an age-adjusted SHR of dementia associated of 2.17 (95\% CI 1.67-2.82). The age-adjusted rates of dementia per 1000 person-years for men with and without bipolar disorder were 32.9 (95\% CI 25.8-41.9) and 14.3 (95\% CI 13.9-14.8) respectively. We stratified these annual rates by age at the time of diagnosis of dementia: 65-70, 70-75, 75-80, 80-85, 85-90 and 90-95 years, which for men without bipolar disorder were $3.0 \%$ (95\% CI 2.4-3.6), 5.1\% (95\% CI 4.7-5.6), $11.5 \%$ 


\begin{tabular}{|c|c|c|c|}
\hline & Population $(n=37768), n$ (\%) & Bipolar disorder $(n=256), n(\%)$ & Odds ratio $(95 \% \mathrm{Cl})$ for bipolar disorder \\
\hline \multicolumn{4}{|l|}{ Age group, years } \\
\hline $65-69$ & $13349(35.3)$ & $94(38.0)$ & 1 (1) \\
\hline 70-74 & 13021 (34.5) & $97(37.6)$ & $1.06(0.80-1.41)$ \\
\hline 75-79 & $8553(22.6)$ & $53(20.5)$ & $0.88(0.63-1.23)$ \\
\hline$\geqslant 80$ & $2845(7.5)$ & $12(3.8)$ & $0.60(0.33-1.09)$ \\
\hline Alcohol use disorder & $1480(3.9)$ & $43(16.8)$ & $5.07(3.64-7.06)$ \\
\hline Other substance use disorder & 5995 (15.9) & $62(24.2)$ & $1.70(1.28-2.27)$ \\
\hline
\end{tabular}

(95\% CI 10.9-12.1), 23.8\% (95\% CI 22.7-24.9), 44.1\% (95\% CI 41.5-46.8) and 59.9\% (95\% CI 51.7, 69.5), whereas for men with bipolar disorder rates were $15.3 \%$ (95\% CI 4.9-47.3), 25.1\% (95\% CI 15.1-41.7), 33.4\% (95\% CI 22.2-50.3), 51.1\% (95\% CI 33.0-79.3) and 41.6\% (95\% CI 15.6-110.7). There were no participants with bipolar disorder in the age group 90-95 years.

The mean age of the first recorded diagnosis of bipolar disorder in this population was 59.7 years (s.d. $=8.9$, range: $37.8-81.7$ ) (see online Fig. DS1 for HR and SHR of dementia by age of bipolar disorder diagnosis). We also investigated the association between time lived with bipolar disorder (in years) and subsequent risk of dementia. These results are summarised in Fig. 2 and show that the HR of dementia was similarly high among men with recent ( $<5$ years) and long-standing history of bipolar disorder $(\geqslant 15$ years) compared with participants without bipolar disorder.

The age-adjusted mortality HR of men with bipolar disorder was 1.51 (95\% CI $1.28-1.77)$, and the mortality rates per 1000 person-years were 69.7 (95\% CI 59.5-91.7) and 49.4 (95\% CI 48.7-50.2) for men with and without bipolar disorder respectively (online Fig. DS2). Further adjustment of the analyses for pastrecorded history of alcohol or substance use disorder had minimal effect on the results ( $\mathrm{HR}=1.32,95 \% \mathrm{CI} 1.13-1.55)$. Table 2 shows the principal causes of death in the population and among participants with bipolar disorder. Competing risk regression showed that bipolar disorder was associated with increased SHR of suicide $(\mathrm{SHR}=13.43,95 \%$ CI 5.35-33.73), accidents $(\mathrm{SHR}=2.78,95 \%$ CI 1.14-6.75), pneumonia or influenza

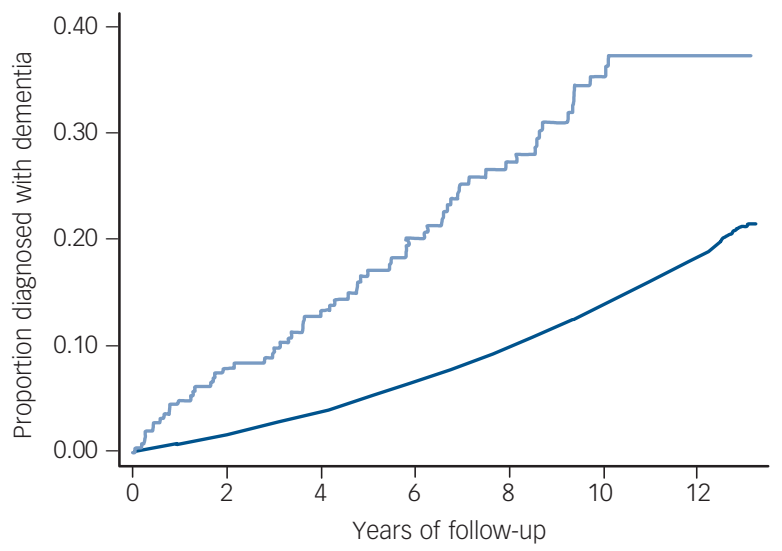

Number at risk

$\begin{array}{llllllll}\text { Not bipolar } & 37512 & 34762 & 31716 & 28517 & 25382 & 22165 & 7028\end{array}$ $\begin{array}{llllllll}\text { Bipolar } & 256 & 220 & 192 & 164 & 140 & 106 & 34\end{array}$

Fig. 1 Cumulative hazard of dementia during a follow-up period of 13 years for older men with (light blue line) and without (dark blue line) bipolar disorder.

The age-adjusted hazard ratio of dementia associated with the diagnosis of bipolar disorder was $2.58(95 \% \mathrm{Cl} 2.02-3.30)$. The age-adjusted sub-hazard ratio of dementia was 2.17 (95\% Cl 1.67-2.82), with death used as a competing risk. The age-adjusted rates of dementia per 1000 person-years for men with and without bipolar disorder were $32.9(95 \% \mathrm{Cl} 25.8-41.9)$ and $14.3(95 \% \mathrm{Cl} 13.9-14.8)$ respectively.
(SHR $=3.66,95 \%$ CI 1.81-7.40), and diseases of the liver and digestive system ( $\mathrm{SHR}=2.02,95 \% \mathrm{CI} 1.00-4.06)$. The age-adjusted suicide rates per 100000 person-years among men with and without bipolar disorder were 229.3 (95\% CI 95.4-550.8) and 15.3 (95\% CI 11.7-20.0) respectively. Similarly, death by accident occurred at a rate of 229.3 (95\% CI 95.4-550.8) and 73.4 (95\% CI 64.983.0) per 100000 person-years for men with and without bipolar disorder respectively. Mortality data stratified according to the age at onset of bipolar disorder and duration of illness are reported in online Tables DS1 and DS2.

\section{Post hoc analyses}

We completed a series of additional analyses to clarify whether the excess risk of dementia and death associated with bipolar disorder could be attributed to the inclusion of false negative cases of dementia in the sample. We re-ran our Cox regression models after excluding participants who met criteria for the diagnosis of dementia within the first 2, 3 and 4 years of follow-up. The respective age-adjusted HRs of dementia associated with the diagnosis of bipolar disorder were 2.15 (95\% CI 1.61-2.87), 2.22 (95\% CI 1.64-2.99) and 2.03 (95\% CI 1.45-2.83). In the same way, we examined the mortality HR associated with the diagnosis of bipolar disorder after excluding participants who died within the first 2, 3 and 4 years of follow-up. The respective mortality HRs were 1.52 (95\% CI 1.28-1.81), 1.56 (1.30-1.86) and 1.56 (1.29-1.89). Finally, we examined the mortality HR associated with the diagnosis of bipolar disorder after excluding from the analyses participants who received a diagnosis of dementia within

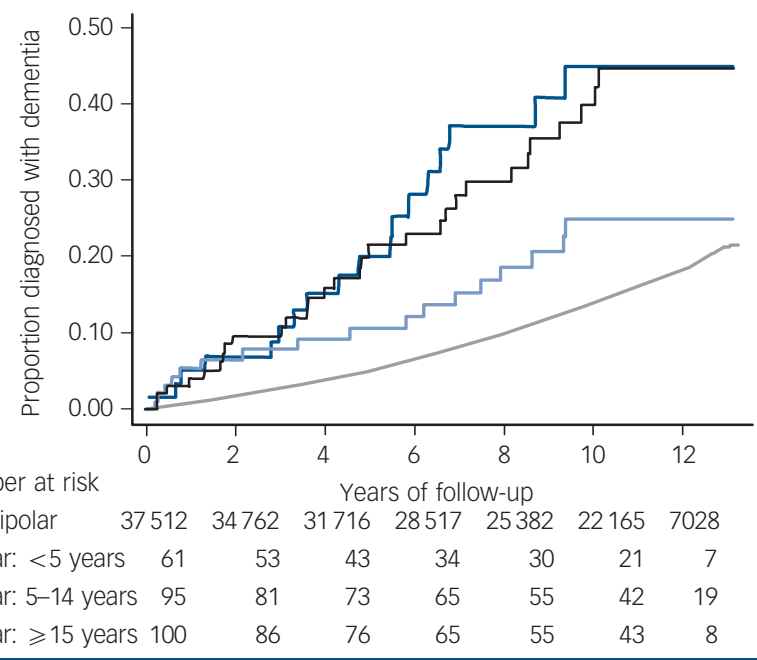

Fig. 2 Age-adjusted cumulative hazard of dementia according to the time lived with the diagnosis of bipolar disorder

Not bipolar (grey line - reference), $<5$ years (dark blue line; hazard ratio (HR) 3.23, $95 \% \mathrm{Cl} 2.03-5.14$ ), $5-14$ years (pale blue line; $\mathrm{HR}=1.71,95 \% \mathrm{Cl} 1.06-2.76$ ), $\geqslant 15$ years (black; HR=3.09, 95\% Cl 2.16-4.43). The respective age-adjusted sub-hazard ratios of dementia were 2.69 (95\% $\mathrm{Cl} 1.62-4.48), 1.41$ (95\% $\mathrm{Cl} 0.86-2.31)$ and 2.68 (95\% $\mathrm{Cl} 1.81-3.95)$, with death used as a competing risk. 
Table 2 Principal causes of death in community-representative sample of older men and among those with bipolar disorder over a follow-up period of 13 years

\begin{tabular}{|c|c|c|c|c|}
\hline & $\begin{array}{c}\text { Total sample } \\
(n=37768) \\
n(\%)\end{array}$ & $\begin{array}{c}\text { Bipolar disorder } \\
\quad(n=256) \\
n(\%)\end{array}$ & $\begin{array}{l}\text { Mortality sub-hazard } \\
\text { ratio for bipolar } \\
\text { disorder }\end{array}$ & $95 \% \mathrm{Cl}$ \\
\hline Alive & $20433(54.1)$ & $104(40.6)$ & 1 & Reference \\
\hline \multicolumn{5}{|l|}{ Causes of death } \\
\hline suicide & $58(0.1)$ & $5(1.9)$ & 13.43 & $5.35-33.73$ \\
\hline Accident & $260(0.7)$ & $5(1.9)$ & 2.78 & $1.14-6.75$ \\
\hline Infection & $314(0.8)$ & $3(1.2)$ & 1.35 & $0.43-4.20$ \\
\hline Cancer & $4484(11.9)$ & $41(16.0)$ & 1.33 & $0.97-1.81$ \\
\hline Diseases of the heart & $4273(11.3)$ & $26(10.2)$ & 0.85 & $0.58-1.26$ \\
\hline Diseases of the brain & $3950(10.5)$ & $31(12.1)$ & 1.12 & $0.79-1.59$ \\
\hline Vascular diseases & $532(1.4)$ & $6(2.3)$ & 1.61 & $0.72-3.62$ \\
\hline Pneumonia or influenza & $319(0.8)$ & $8(3.1)$ & 3.66 & $1.81-7.40$ \\
\hline Respiratory diseases & $1636(4.3)$ & $10(3.9)$ & 0.86 & $0.46-1.60$ \\
\hline Liver and digestive diseases & $568(1.5)$ & $8(3.1)$ & 2.02 & $1.00-4.06$ \\
\hline Kidney diseases & $421(1.1)$ & $4(1.6)$ & 1.35 & $0.50-3.63$ \\
\hline Other causes & Men's S520 (1.4) & $5(1.9)$ & 1.36 & $0.56-3.29$ \\
\hline
\end{tabular}

the first 2, 3 and 4 years of follow-up - the respective mortality HRs were 1.45 (95\% CI $1.21-1.74), 1.49$ (95\% CI $1.23-1.81)$ and 1.48 (95\% CI 1.21-1.83).

As several unmeasured factors could have confounded the association between bipolar disorder and dementia/mortality, we conducted supplementary analyses with a subsample of men for whom lifestyle and clinical data were collected between 1996 and 1998 by means of a self-report questionnaire. Details about this cohort have been reported elsewhere. ${ }^{19}$ The sample consisted of 12147 participants free of dementia, of whom 65 had bipolar disorder $(0.5 \%)$. Other relevant measured factors included place of birth (Australia $v$. elsewhere), marital status (married $v$. not married), education (complete $v$. incomplete high school), physical activity $(<150 v . \geqslant 150 \mathrm{~min} /$ week of vigorous physical activity), smoking (never, past or current), alcohol consumption (never, past, $<14 \mathrm{~g} /$ week, $14-27 \mathrm{~g} /$ week, $\geqslant 28 \mathrm{~g} /$ week), body mass index $\left(<18.5 \mathrm{~kg} / \mathrm{m}^{2}, 18.5-25.9 \mathrm{~kg} / \mathrm{m}^{2}, 25-29.9 \mathrm{~kg} / \mathrm{m}^{2}, \geqslant 30 \mathrm{~kg} / \mathrm{m}^{2}\right)$, history or treatment for diabetes or hypertension, and past heart attack or stroke. We included these variables, as well as age, in a Cox regression model to investigate the effect of history of bipolar disorder on the risk of dementia - the relevant adjusted HR was 2.52 (95\% CI 1.54-4.15). Similarly, the adjusted mortality hazard associated with the diagnosis of bipolar disorder was 1.40 (95\% CI $1.01-1.95)$.

\section{Discussion}

We believe this is the first longitudinal study of older adults with bipolar disorder derived from a community-representative sample. The results showed that dementia and death accrue with greater frequency among older men with than without bipolar disorder. The diagnosis of bipolar disorder was associated with more than double the risk of dementia over 13 years and with a $50 \%$ increase in mortality. This excess risk could not be attributed to confounding due to age or use of substances. We also observed that the risk of dementia was highest for men with recent $(<5$ years) and long-standing history of bipolar disorder ( $\geqslant 15$ years), and that death by suicide, accidents, pneumonia or influenza, and diseases of the digestive system were more frequent among older men with than without bipolar disorder. Finally, we found that the causes of death varied according to the age at onset and duration of bipolar disorder, with diseases of the brain being more frequent among men with $<5$ years history of bipolar disorder or with illness onset in their 70 s or later.

\section{Strengths and limitations}

This study has the merit of having used exposure and outcome data for the entire male population of older Australian citizens living in Perth. We acknowledge, however, that older adults living in residential care facilities and those who were not citizens were not approached (about $20 \%$ of this population). ${ }^{20}$ We are not aware of any evidence indicating that the clinical outcomes of older non-Australian citizens are different from their Australian peers. Another important feature of a longitudinal study such as this is that follow-up data were available through WADLS for all participants, as the internal and external migratory movement of older Western Australians is negligible. ${ }^{21}$

We concede that the diagnosis of bipolar disorder relied on administrative data, which only became available in 1966. Consequently, participants who were in contact with the health services before 1966, but not later, would not have been identified as cases, although the chronic and recurring nature of bipolar disorder would most likely have ensured identification at a later stage. Nonetheless, if false negative cases were present in the 'non-bipolar' sample, this would have decreased the power of the study. The other consequence of the 1966 starting date for WADLS is that it could have biased our estimates of age at onset and duration of illness. We attempted to address this issue by stratifying time-related data into groups (e.g. $<5$ years, 5-14 years and $\geqslant 15$ years of illness), bearing in mind that 30 years of data had already been accumulated by the time we initiated follow-up. We are also reassured by the fact that the lifetime prevalence of bipolar disorder in our sample $(0.7 \%)$ is consistent with that of other epidemiological surveys that used structured interviews and face-to-face assessments. ${ }^{22}$

Like bipolar disorder, the diagnosis of dementia relied on data recorded in WADLS. We suspect that many older adults with mild dementia remain undiagnosed or are managed in primary care, which could have led to underestimation of the true rate of dementia in the sample, although our incidence rate figures were within the range of those reported by others. ${ }^{23}$ Nevertheless, if we accept that some false negative cases of dementia were present, the calculation of risk associated with bipolar disorder would only be affected if the distribution of these cases in the population were not random. It is conceivable that medical specialists attuned to 
the assessment of cognitive function and dementia (such as geriatric psychiatrists) review older men with bipolar disorder more often and, thus, establish a diagnosis of dementia for these patients earlier than otherwise would have been the case. Such bias could have potentially inflated the risk ratio of dementia among older men with bipolar disorder compared with the rest of the population. We are unable to dismiss such a possibility outright, but our post hoc analyses showed that the observed risk estimates did not change substantially when we excluded from the analyses participants who had developed dementia during the first 4 years of follow-up (i.e. possible undiagnosed prevalent cases of dementia at study entry). Prior studies that had access to control groups with other mental disorders ${ }^{8}$ or medical illnesses ${ }^{24}$ have also reported a relative excess of cases of dementia among people with past diagnosis of bipolar disorder, thereby suggesting that detection bias may be insufficient to explain the association between bipolar disorder and dementia.

We also concede that several unmeasured factors could have confounded the association between bipolar disorder, dementia and death, such as physical activity, smoking, education, marital status and comorbidities. These data were available for a subsample of 12147 of our participants - the adjusted post hoc models yielded results that were similar to those observed for the entire sample, which suggests that the link between bipolar disorder and dementia and death cannot be easily attributed to lifestyle and clinical factors, although residual confounding remains a possibility.

The relatively small number of participants with bipolar disorder introduced some imprecision in the calculation of the risk (and rates) associated with different causes of death, as some of the events were uncommon (such as suicides). For this reason, these results would be better viewed as tentative. Finally, we acknowledge that the study was limited to men and it is unclear whether our findings would be equally applicable to women.

\section{Interpretation}

Our data showed that older men with past diagnosis of bipolar disorder are at increased risk of dementia and that this risk is greatest for those with long-standing and with recent history of bipolar disorder. The association between a recent diagnosis of bipolar disorder (i.e. $<5$ years) and dementia is consistent with the possibility that changes in the regulation of mood later in life may represent, in at least some cases, an early clinical expression of neurodegenerative processes that will ultimately lead to the diagnosis of dementia. The doubling in the hazard of death due to diseases of the brain among those in their $70 \mathrm{~s}$ or $80 \mathrm{~s}$ as well as among those with bipolar disorder of $<5$ years duration lends support to this interpretation.

An early case series of 92 older adults admitted to hospital with an episode of mania found that 22 of them (24\%) showed evidence of brain disease consistent with dementia, Parkinson's or cerebrovascular diseases. ${ }^{25}$ Others later reported that 8 of 25 older people $(32 \%)$ treated for mania developed cognitive impairment over a follow-up period of 5-7 years, ${ }^{26}$ whereas recent case reports have highlighted the potential overlap between some cases of bipolar disorder and frontotemporal dementia. ${ }^{27,28}$

There is evidence that people with bipolar disorder lose more grey matter volume in the medial temporal cortex over 4 years than controls, and that this loss is correlated with the number of affective episodes. ${ }^{29}$ In this case, long-standing and recurring bipolar disorder would be expected to be associated with increased risk of cognitive decline, as suggested by others ${ }^{7}$ and corroborated by our findings. The pathophysiological processes underpinning these changes remain unclear, but some have argued that inflammation ${ }^{30}$ and cardiovascular risk factors may be involved. ${ }^{31}$ In addition, there have been suggestions that people with bipolar disorder may develop dementia or die earlier because of accelerated ageing, although a meta-analysis of studies of telomere length that included people with bipolar disorder found no difference between patients and controls. ${ }^{32}$

Existing observational data indicate a lower risk of dementia among people with bipolar disorder who are issued a prescription for treatment with lithium, ${ }^{33}$ with a subsequent study reporting that prolonged treatment may be required to consolidate this benefit. ${ }^{34}$ These studies found no evidence of benefit associated with the use of other mood stabilisers, and high-quality corroboration from randomised controlled trials remains sparse. A 13-week placebo-controlled trial of 71 patients with Alzheimer's disease found that treatment with lithium was not associated with cognitive gains, ${ }^{35}$ although the 12 -month treatment of 21 adults with amnestic mild cognitive impairment led to a decrease in the concentration of phosphorylated tau in the cerebrospinal fluid and a trend for improved cognitive performance compared with the 20 placebo-treated participants. ${ }^{36}$ Hence, treatment with lithium could contribute to prevent dementia, but a sufficiently powered randomised placebo-controlled trial would be required to test this hypothesis.

Several past surveys have reported that bipolar disorder is associated with higher mortality due to general medical illnesses, ${ }^{10,11,37}$ but relevant data for older adults with bipolar disorder have not been reported. Self-harm behaviour, including suicide, is more frequent among older adults with affective disorders than in the general community. ${ }^{38}$ Our data confirmed the excess mortality due to suicide and accidents among older men with bipolar disorder, although the number of individuals affected was small. It is possible that certain lifestyle practices associated with bipolar disorder may also contribute to decrease survival (e.g. excessive use of substances), but other factors may play a part. Investigations have suggested that chronic inflammation drives some of the neurocognitive changes associated with bipolar disorder, ${ }^{39}$ and may also increase the long-term risk of death due to infections, including influenza and pneumonia. ${ }^{40}$ Moreover, the use of mood stabilisers, antipsychotics, antidepressants and benzodiazepines could contribute to increase cognitive impairment and mortality among older adults with bipolar disorder. ${ }^{41-43}$

In summary, this large community-based cohort study of older men showed that bipolar disorder is associated with increased risk of dementia and with premature death. The pathways linking bipolar disorder to these clinical outcomes are yet to be established, but currently available evidence suggests that some of them may be amenable to change, in which case prevention might not only be desirable but also feasible.

Osvaldo P. Almeida, PhD, School of Psychiatry \& Clinical Neurosciences, University of Western Australia, Perth, WA Centre for Health \& Ageing of Centre for Medical Research, Harry Perkins Institute of Medical Research, Perth, and Department of Psychiatry, Royal Perth Hospital, Perth; Kieran McCaul, PhD, WA Centre for Health \& Ageing of Centre for Medical Research, Harry Perkins Institute of Medical Research, Perth; Graeme J. Hankey, MD, School of Medicine and Pharmacology, University of Perth; Graeme J. Hankey, MD, School of Medicine and Pharmacology, University of
Western Australia, Perth, and Department of Neurology, Sir Charles Gairdner Hospital, Perth; Bu B. Yeap, PhD, School of Medicine and Pharmacology, University of Western Australia, Perth, and Department of Endocrinology, Fiona Stanley Hospital, Perth; Jonathan Golledge, MChir, Queensland Research Centre for Peripheral Vascular Disease, College of Medicine and Dentistry, James Cook University, Townsville, and Department of Vascular and Endovascular Surgery, The Townsville Hospital, Townsville; Leon Flicker, PhD, WA Centre for Health \& Ageing of Centre for Medical Research, Harry Perkins Institute of Medical Research, Perth, School of Medicine and Pharmacology, University of Western Australia, Perth, and Department of Geriatric Medicine, Royal Perth Hospital, Perth, Australia

Correspondence: Professor Osvaldo P. Almeida, School of Psychiatry \& Clinical Neurosciences (M573), University of Western Australia, 35 Stirling Highway, Crawley, Perth, WA 6009, Australia. Email: osvaldo.almeida@uwa.edu.au

First received 13 Dec 2015, accepted 1 Feb 2016 


\section{References}

1 DelBello MP, Hanseman D, Adler CM, Fleck DE, Strakowski SM. Twelvemonth outcome of adolescents with bipolar disorder following first hospitalization for a manic or mixed episode. Am J Psychiatry 2007; 164 582-90

2 Mora E, Portella MJ, Forcada I, Vieta E, Mur M. Persistence of cognitive impairment and its negative impact on psychosocial functioning in lithium-treated, euthymic bipolar patients: a 6-year follow-up study. Psychol Med 2013; 43: 1187-96.

3 Bourne C, Aydemir O, Balanza-Martinez V, Bora E, Brissos S, Cavanagh JT, et al. Neuropsychological testing of cognitive impairment in euthymic bipola disorder: an individual patient data meta-analysis. Acta Psychiatr Scand 2013; 128: 149-62.

4 Samame C, Martino DJ, Strejilevich SA. A quantitative review of neurocognition in euthymic late-life bipolar disorder. Bipolar Disord 2013; 15: $633-44$.

5 Martinez-Aran A, Vieta E, Torrent C, Sanchez-Moreno J, Goikolea JM Salamero $\mathrm{M}$, et al. Functional outcome in bipolar disorder: the role of clinical and cognitive factors. Bipolar Disord 2007; 9: 103-13.

6 Schouws SN, Comijs HC, Stek ML, Dekker J, Oostervink F, Naarding P, et al. Cognitive impairment in early and late bipolar disorder. Am J Geriatr Psychiatry 2009; 17: 508-15.

7 Kessing LV, Andersen PK. Does the risk of developing dementia increase with the number of episodes in patients with depressive disorder and in patients with bipolar disorder? J Neurol Neurosurg Psychiatry 2004; 75: 1662-6.

8 Kessing LV, Olsen EW, Mortensen PB, Andersen PK. Dementia in affective disorder: a case-register study. Acta Psychiatr Scand 1999; 100: 176-85.

9 Wu KY, Chang CM, Liang HY, Wu CS, Chia-Hsuan Wu E, Chen $\mathrm{CH}$, et al. Increased risk of developing dementia in patients with bipolar disorder: a nested matched case-control study. Bipolar Disord 2013; 15: 787-94.

10 Kessing LV, Vradi E, Andersen PK. Life expectancy in bipolar disorder. Bipola Disord 2015; 17: 543-8.

11 Kessing LV, Vradi E, McIntyre RS, Andersen PK. Causes of decreased life expectancy over the life span in bipolar disorder. J Affect Disord 2015; 180 $142-7$.

12 Almeida OP, Hankey GJ, Yeap BB, Golledge J, Norman PE، Flicker L. Mortality among people with severe mental disorders who reach old age: a longitudinal study of a community-representative sample of 37,892 men. PLOS One 2014; 9: e111882.

13 Crump C, Sundquist K, Winkleby MA, Sundquist J. Comorbidities and mortality in bipolar disorder: a Swedish national cohort study. JAMA Psychiatry 2013; 70: 931-9.

14 Holman CD, Bass AJ, Rosman DL, Smith MB, Semmens JB, Glasson EJ, et al. A decade of data linkage in Western Australia: strategic design, applications and benefits of the WA data linkage system. Aust Health Rev 2008; 32 : 766-77.

15 World Health Organization. International Classification of Diseases, Eighth Revision (ICD-8). WHO, 1965

16 World Health Organization. International Classification of Diseases, Ninth Revision (ICD-9). WHO, 1979.

17 World Health Organization. International Classification of Diseases, Tenth Revision (ICD-10). WHO, 1999.

18 Jablensky AV, Morgan V, Zubrick SR, Bower C, Yellachich LA. Pregnancy, delivery, and neonatal complications in a population cohort of women with schizophrenia and major affective disorders. Am J Psychiatry 2005; 162: 79-91.

19 Norman PE, Flicker L, Almeida OP, Hankey GJ, Hyde Z, Jamrozik K. Cohort Profile: The Health In Men Study (HIMS). Int J Epidemiol 2009; 38: 48-52.

20 McLennan W. Australian Social Trends 1996. Canberra: Australia (Report Catalogue number 4102.0). Australian Bureau of Statistics; 1996.

21 Australian Bureau of Statistics. 3412.0 - Migration, Australia, 2013-14. Australian Bureau of Statistics, 2015 (available at http://www.abs.gov.au/ ausstats/abs@.nsf/mf/3412.0/ (last accessed 4 Feb 2016).
22 Kessler RC, Berglund P, Demler O, Jin R, Merikangas KR, Walters EE. Lifetime prevalence and age-of-onset distributions of DSM-IV disorders in the National Comorbidity Survey Replication. Arch Gen Psychiatry 2005; 62: 593-602.

23 Vardarajan BN, Faber KM, Bird TD, Bennett DA, Rosenberg R, Boeve BF, et al. Age-specific incidence rates for dementia and Alzheimer disease in NIA-LOAD/NCRAD and EFIGA families: National Institute on Aging Genetics Initiative for Late-Onset Alzheimer Disease/National Cell Repository for Alzheimer Disease (NIA-LOAD/NCRAD) and Estudio Familiar de Influencia Genetica en Alzheimer (EFIGA). JAMA Neurol 2014; 71: 315-23.

24 Kessing LV, Nilsson FM. Increased risk of developing dementia in patients with major affective disorders compared to patients with other medical illnesses. J Affect Disord 2003; 73: 261-9.

25 Stone K. Mania in the elderly. Br J Psychiatry 1989; 155: 220-4.

26 Dhingra U, Rabins PV. Mania in the elderly: a 5-7 year follow-up. J Am Geriatr SOC 1991; 39: 581-3.

27 Cerami C, Marcone A, Galimberti D, Villa C, Scarpini E, Cappa SF. From genotype to phenotype: two cases of genetic frontotemporal lobar degeneration with premorbid bipolar disorder. J Alzheimers Dis 2011; 27 791-7.

28 Floris G, Borghero G, Cannas A, Stefano FD, Murru MR, Corongiu D, et al. Bipolar affective disorder preceding frontotemporal dementia in a patient with C9ORF72 mutation: is there a genetic link between these two disorders? J Neurol 2013; 260: 1155-7.

29 Moorhead TW, McKirdy J, Sussmann JE, Hall J, Lawrie SM, Johnstone EC, et al. Progressive gray matter loss in patients with bipolar disorder. Biol Psychiatry 2007; 62: 894-900.

30 Bauer IE, Pascoe MC, Wollenhaupt-Aguiar B, Kapczinski F, Soares JC. Inflammatory mediators of cognitive impairment in bipolar disorder. J Psychiatr Res 2014; 56: 18-27.

31 Schouws SN, Stek ML, Comijs HC, Beekman AT. Risk factors for cognitive impairment in elderly bipolar patients. J Affect Disord 2010; 125: 330-5.

32 Colpo GD, Leffa DD, Kohler CA, Kapczinski F, Quevedo J, Carvalho AF. Is bipolar disorder associated with accelerating aging? A meta-analysis of telomere length studies. J Affect Disord 2015; 186: 241-8.

33 Kessing LV, Sondergard L, Forman JL, Andersen PK. Lithium treatment and risk of dementia. Arch Gen Psychiatry 2008; 65: 1331-5.

34 Kessing LV, Forman JL, Andersen PK. Does lithium protect against dementia? Bipolar Disord 2010; 12: 87-94.

35 Hampel H, Ewers M, Burger K, Annas P, Mortberg A, Bogstedt A, et al. Lithium trial in Alzheimer's disease: a randomized, single-blind, placebocontrolled, multicenter 10-week study. J Clin Psychiatry 2009; 70: 922-31.

36 Forlenza OV, Diniz BS, Radanovic M, Santos FS, Talib LL, Gattaz WF. Disease-modifying properties of long-term lithium treatment for amnestic mild cognitive impairment: randomised controlled trial. Br J Psychiatry 2011; 198: 351-6.

37 Roshanaei-Moghaddam B, Katon W. Premature mortality from general medical illnesses among persons with bipolar disorder: a review. Psychiatr Serv 2009; 60: 147-56

38 Lawrence D, Almeida OP, Hulse GK, Jablensky AV, Holman CD. Suicide and attempted suicide among older adults in Western Australia. Psychol Med 2000; 30: 813-21.

39 Berk M, Kapczinski F, Andreazza AC, Dean OM, Giorlando F, Maes M, et al. Pathways underlying neuroprogression in bipolar disorder: focus on inflammation, oxidative stress and neurotrophic factors. Neurosci Biobehav Rev 2011; 35: 804-17.

40 Ritchie SC, Würtz P, Nath AP, Abraham G, Havulinna AS, Fearnley LG, et al. The biomarker GlycA is associated with chronic inflammation and predicts long-term risk of severe infection. Cell Systems 2015; 1: 293-301.

41 Almeida OP, Alfonso H, Hankey GJ, Flicker L. Depression, antidepressant use and mortality in later life: the Health In Men Study. PLOS One 2010; 5 e11266.

42 Wang PS, Schneeweiss S, Avorn J, Fischer MA, Mogun $\mathrm{H}$, Solomon $\mathrm{DH}$, et al. Risk of death in elderly users of conventional vs. atypical antipsychotic medications. N Engl J Med 2005; 353: 2335-41.

43 Vinkers DJ, Gussekloo J, van der Mast RC, Zitman FG, Westendorp RG. Benzodiazepine use and risk of mortality in individuals aged 85 years or older. JAMA 2003; 290: 2942-3. 